\title{
The coupled effects of protostellar outflows, radiation feedback, magnetic fields and turbulence on the formation of massive stars and Orion-like clusters
}

\author{
Richard I. Klein ${ }^{1,2}$ \\ ${ }^{1}$ Department of Astronomy, University of California, Berkeley, CA 94720, USA, \\ email: rklein@astron.berkeley.edu \\ ${ }^{2}$ Lawrence Livermore National Laboratory, \\ Livermore, CA 95550, USA
}

\begin{abstract}
Feedback processes from massive stars plays a critical role in their formation, destroy the molecular clouds in which they are born and shape the evolution of galaxies. In this talk I will discuss our recent 3D AMR simulations that are the first to include the coupled feedback effects of protostellar outflows combined with protostellar heating and radiation pressure feedback and magnetic fields, in a single computation and their effects on the infalling dusty gas in the surrounding environs of the accreting core envelope. These simulations will address the detailed effects of feedback on the formation of high mass stars and massive clusters with implications for the IMF.
\end{abstract}

Keywords. star formation, stars: high mass, MHD, radiative transfer, turbulence, outflows

\section{Introduction}

The problem of high mass star formation is complicated by the several physical feedback processes that are at play and may be critically important in determining the final stellar mass of the star which have been observed in mass ranges of $120-300 M_{\odot}$. Strong radiative forces communicated to the dusty accretion envelope surrounding the central protostar oppose the force of gravity of the accreting gas as it attempts to make its way onto the accretion disk surrounding the protostar and make its final plunge onto the star as it builds in mass. A delicate balance between these opposing forces results in a tug of war. To further complicate the fate of the final mass of the star, strong protostellar outflows, magnetic fields and ionizing radiation from the central object also contribute to the balance. See Klein (2008) for a detailed discussion of the physical processes. Through the use of highly resolved radiation-magnetohydrodynamic adaptive mesh refinement (AMR) simulations, we can assess the effects of the individual feedback processes and the interaction of the coupled processes on high mass star formation and formation of massive clusters. Our simulations are performed using the state-of-the-art code we have developed at Berkeley, ORION, which is a parallelized 3D AMR Magneto-Rad-Hydro code (Truelove et al. 1998; Klein 1999; Krumholz et al. 2007c; Cunningham et al. 2012).

\section{Feedback effects in high mass star formation and clusters}

In a series of papers, Krumholz, Klein \& McKee (2007a,b,c), have shown the first 3D simulations incorporating the relevant physical processes of radiation feedback to follow the formation of a massive star from the collapse of its embryonic turbulent core 

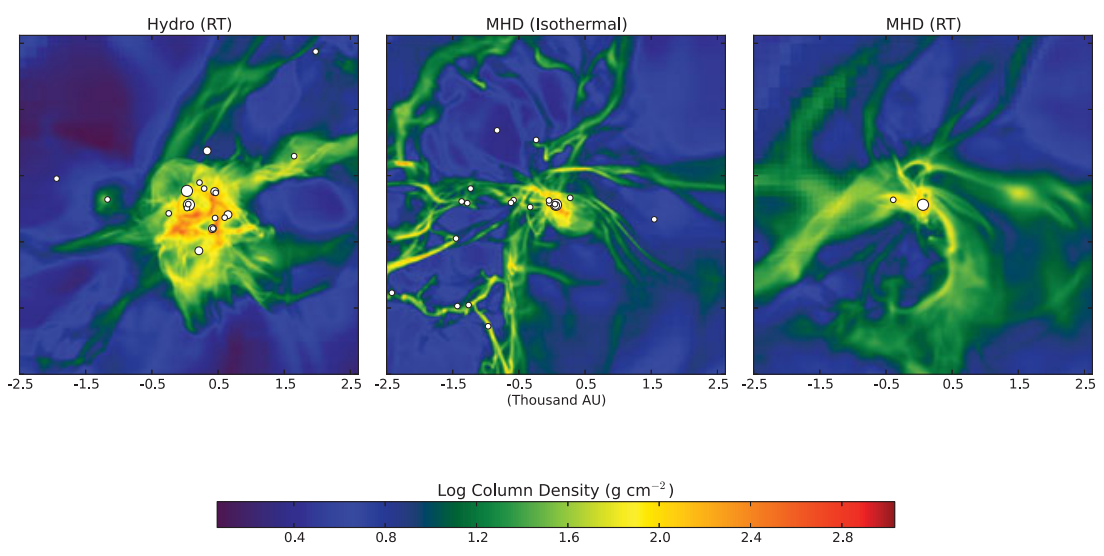

Figure 1. Column density through the simulation volume for simulations with Hydro with radiative transfer (HR); MHD with no radiative transfer in the isothermal approximation (BI) and MHD with radiative transfer (BR). Projections are taken along the $\mathrm{x}$ direction and the initial magnetic field is oriented in the positive z direction. Star particles are portrayed as white circles. The smallest circles represent stars with masses between $0.05 M_{\odot}$ and $1.0 M_{\odot}$; the next size up represents masses between $1.0 M_{\odot}$ and $8.0 M_{\odot}$, and the largest size represents stars with $\geqslant 8.0 M_{\odot}$ in each simulation. The figure is zoomed in to show the central $5000 \mathrm{AU}$ around the most massive star in each simulation.

down to the formation of the massive protostar. This work has led to solutions to three longstanding problems in massive star formation theory: fragmentation, angular momentum transport, and radiation pressure. It was shown that the radiation emitted by a accreting high-mass stars can heat up clouds to the point where fragmentation is suppressed (Krumholz, Klein, \& McKee 2007a), that angular momentum can be transported very rapidly by large-scale gravitational instabilities in the accretion disks around massive stars (Krumholz, Klein, \& McKee 2007b), and that radiation pressure does not halt accretion, because radiation-hydrodynamic instabilities, essentially radiation driven Rayleigh Taylor instabilities, reshape the stellar radiation field, beaming it away from the bulk of the incoming gas (Krumholz et al. 2009). These simulations also demonstrated convincingly that the disks around massive stars inevitably fragment to produce massive companions, explaining the observed ubiquity of multiple systems among massive stars. The next important feedback effect on the formation of massive star is the protostellar outflows that interact with the still accreting protostar. In recent work (Cunnngham et al. 2011), we made the first simulations to include the feedback effects of protostellar outflows with protostellar radiative heating and radiation pressure. It was shown that feedback from protostellar outflows resulted in the the diminishing of radiative heating and outward radiation force exerted on the protostellar disk and infalling gas thereby reducing the Eddington radiation pressure barrier to high-mass star formation. We also found that radiation focusing in the direction of outflow cavities is sufficient to prevent the formation of radiation pressure-supported circumstellar gas bubbles, in contrast to models which neglect protostellar outflow feedback. In recent work (Myers et al. 2012 submitted), we present the first results of 3-dimensional, AMR radiation-magnetohydrodynamic simulations that treat the dust processed radiation from protostars in the flux-limiteod diffusion approximation. The question of how isolated massive cores fragment is a crucial question for any theory of star formation in which the initial mass function (IMF) is set in the gas phase, e.g. the turbulent fragmentation scenario (Padoan \& Nordund 2002). In Figure 1 we note that simulations with radiation alone (HR) or magnetic fields alone (BI) result in multiple fragmentation with a low mass cluster of stars and a 
single high mass star $\left(\sim 22 M_{\odot}\right)$. Simulations that have both radiative transfer coupled to magnetic fields produce a dramatically different result. As Myers et al. (2012) show, considering the thermodynamics of the core, both magnetic fields and radiation feedback are necessary to fully suppress multiple star formation leaving a single high mass star of $\sim 20 M_{\odot}$ and a low mass star. The coupled effects of magnetic fields and radiation (BR) are critical in that magnetic fields alone raise the effective temperature in the core in low density gas which suppresses fragmentation in diffuse gas far from central stars in the core. Alternatively, radiation alone raises the effective temperature in high density gas thus suppressing fragmentation in dense gas near the star. However, the interaction of both magnetic fields and radiation together suppress fragmentation far more effectively than either alone since they cover the domain of the core that the other one misses. Radiation feedback can also have a dramatic effect on the evolution and formation of massive star clusters. Recent work that for the first time includes the feedback effects of radiation, protostellar outflows and turbulence on the formation of a massive star cluster (Krumholz, Klein \& McKee 2011,2012) are the first simulations published to date that reproduces the observed time invariant IMF in a cluster like the ONC that is large enough to contain massive stars, and where the peak of the mass function is determined by a fully self-consistent calculation of gas thermodynamics.

\section{Conclusions}

We have briefly discussed recent 3D AMR simulations that for the first time have treated the coupled effects of radiation, protostellar outflows and magnetic fields and their effects on the formation of individual high mass stars and massive clusters.

\section{Acknowledgements}

Support for this work was provided by: the US Department of Energy at the Lawrence Livermore National Laboratory under contract DE-AC52-07NA27344, NASA through ATFP grant NNX09AK31G and the National Science Foundation through grant AST0908553.

\section{References}

Cunningham, A. J., Klein, R. I., Krumholz, M. R., \& McKee, C. F. 2011, ApJ 740, 107

Klein, R. I. 2008, in Massive Star Formation: Observations Confront Theory, eds. H. Beauther, H. Linz, \& T. Henning, (ASP Conference Series) 38, 306

Klein, R. I. 1999, J. Comp. App. Math. 109, 123

Krumholz, M. R., Klein, R. I., \& McKee, C. F. 2007a, ApJ 656, 959

Krumholz, M. R., Klein, R. I., \& McKee, C. F. 2007b, ApJ 665, 478

Krumholz, M. R., Klein, R. I., \& McKee, C. F. 2007c, ApJ 667, 626

Krumholz, M. R., Klein, R. I., McKee, C. F., Offner, S. S. R., \& Cunningham, A. J. 2009, Science 323,754

Krumholz, M. R., Klein, R. I., \& McKee, C. F. 2011, ApJ 740, 74

Krumholz, M. R., Klein, R. I., \& McKee, C. F. 2012, ApJ 754, 71

Myers, A. T., McKee, C. F., Cunningham, A. J. Klein, R, I., \& Krumholz, M. R. 2012, submitted to $A p J(\operatorname{arXiv} 1211.3467 \mathrm{M})$

Padoan, P. \& Nordlund, A. A. 2002, ApJ 576,870

Truelove, K. J., Klein, R. I., \& McKee, C. F. 1998, ApJ 495, 821 